\title{
Télescope
}

Revue d'analyse comparée en administration publique

\section{L'innovation au sein des entreprises et du gouvernement : se tourner vers l'avenir}

\section{Sandford Borins}

Volume 19, numéro 2, printemps 2013

L'innovation dans le secteur public : au-delà des discours

URI : https://id.erudit.org/iderudit/1023840ar

DOI : https://doi.org/10.7202/1023840ar

Aller au sommaire du numéro

Éditeur(s)

L'Observatoire de l'administration publique

ISSN

1929-3348 (numérique)

Découvrir la revue

Citer cet article

Borins, S. (2013). L'innovation au sein des entreprises et du gouvernement : se tourner vers l'avenir. Télescope, 19(2), 57-70. https://doi.org/10.7202/1023840ar

\section{Résumé de l'article}

Cet article traite des effets de la mise en oeuvre des deux politiques les plus tentaculaires des cinq dernières années - la stimulation économique en réponse à la crise économique mondiale et l'austérité en vue de réduire les déficits gouvernementaux - sur l'innovation dans le secteur public.

L'immédiateté que suppose la stimulation économique a limité la capacité des fonds de relance à soutenir l'innovation dans le secteur public. L'austérité a un effet mixte : une impulsion positive à trouver de nouveaux mécanismes de prestation de services, mais un impact négatif en raison d'une réduction de la marge de manoeuvre utilisée pour soutenir l'innovation. Cet article présente également les résultats d'une étude sur les demandes déposées aux Innovations in American Government Awards 2010. Les résultats montrent une hausse pour des partenariats interorganisationnels, dont les partenariats de cofinancement, comparativement à il y a deux décennies. 


\title{
L'INNOVATION AU SEIN DES ENTREPRISES ET DU GOUVERNEMENT : SE TOURNER VERS L'AVENIR
}

\author{
Par Sandford Borins', Professeur de gestion stratégique, Université de Toronto, \\ Canada et Chercheur agrégé, Harvard Kennedy School, États-Unis \\ borins@utsc.utoronto.ca \\ Traduit de l'anglais
}

RÉSUMÉ Cet article traite des effets de la mise en œuvre des deux politiques les plus tentaculaires des cinq dernières années - la stimulation économique en réponse à la crise économique mondiale et l'austérité en vue de réduire les déficits gouvernementaux - sur l'innovation dans le secteur public. L'immédiateté que suppose la stimulation économique a limité la capacité des fonds de relance à soutenir l'innovation dans le secteur public. L'austérité a un effet mixte : une impulsion positive à trouver de nouveaux mécanismes de prestation de services, mais un impact négatif en raison d'une réduction de la marge de manœuvre utilisée pour soutenir l'innovation. Cet article présente également les résultats d'une étude sur les demandes déposées aux Innovations in American Government Awards 2010. Les résultats montrent une hausse pour des partenariats interorganisationnels, dont les partenariats de cofinancement, comparativement à il y a deux décennies.

ABSTRACT This paper discusses the impact on public sector innovation of the two most significant overarching policy developments in the last five years: economic stimulus in response to the global financial crisis, and austerity intended to reduce government deficits. The former's focus on immediacy limited the extent stimulus funds could support public sector innovation. Austerity had a mixed effect: a positive impetus to find new service delivery mechanism, but a negative impact due to the reduction of slack used to support innovation. The paper also presents results of a study of applications to the 2010 Innovations in American Government Awards that replicates the author's 1998 book Innovating with Integrity. The most significant difference observed is a much greater salience of interorganizational partnerships, including those involving shared funding, now than two decades ago.

La recherche présentée dans cet article a été financée par le ASH Center for Democratic Governance and Innovation de la Harvard Kennedy School. Je tiens à remercier madame Kaylee Chretien pour l'aide à la recherche, les deux experts anonymes pour leurs commentaires et madame Beth Herst pour son travail de révision. Des versions antérieures de cet article ont été présentées à l'Australian National University et à la Roskilde University, au Danemark. 
Désastres financiers, bulles immobilières qui éclatent, taux de chômage élevés qui persistent, crises de la dette, craintes liées au marché monétaire : nous vivons à une époque où les dilemmes macroéconomiques et leurs conséquences consument tant les décideurs politiques et les gestionnaires financiers que les citoyens. Si le débat sur les causes et les responsables de la crise demeure amer et polarisé, les discussions mondiales sur les politiques macroéconomiques n'en ont pas été moins conflictuelles, divisant la population selon les courants idéologiques. La gauche a de façon générale milité pour de plus fortes dépenses gouvernementales, financées soit par un déficit soit par une augmentation de l'imposition des riches; la droite réplique avec des demandes de réductions d'impôts et de dépenses gouvernementales moindres.

Mais il existe une troisième solution, beaucoup moins litigieuse, qui est peut-être née de la crise, mais qui génère des effets qui lui survivront longtemps. Une solution appliquée à une échelle microéconomique, mais pouvant avoir des retombées sur une échelle macroéconomique. Une solution qui cherche à interpeller et à inspirer, plutôt qu'à seulement réagir. Cette solution est l'innovation. La gauche et la droite favorisent toutes deux la mise en œuvre d'idées qui augmenteront la productivité des compagnies et des agences gouvernementales et qui stimuleront la création de nouveaux produits et de nouvelles entreprises. Chaque pays espère qu'une partie importante des nouveaux produits et des nouvelles compagnies s'installeront sur son territoire. Chaque organisme, qu'il soit public ou privé, comprend la valeur de la réinvention pour motiver les employés et attirer les clients, surtout par des temps difficiles.

Bien que notre principale préoccupation soit le secteur public, nous devons nous demander si des leçons peuvent être tirées du succès connu par de grandes sociétés du secteur privé en matière de promotion de l'innovation. Nous traiterons également des incidences qu'a eues la mise en œuvre des deux politiques les plus tentaculaires des cinq dernières années sur l'innovation dans le secteur public, soit la stimulation économique en réponse à la crise économique mondiale et l'austérité en vue de réduire les déficits gouvernementaux. Finalement, nous présenterons des recherches antérieures sur l'innovation au sein du gouvernement et explorerons les effets de ces découvertes sur de futures innovations dans le secteur public.

\section{L'INNOVATION DANS LE SECTEUR PRIVÉ}

Dans le contexte du secteur privé, le concept large d'innovation développé par Schumpeter (2008) englobe l'introduction de nouveaux produits ou l'ajout de nouveaux attributs à des produits existants, les changements dans les processus de production, l'ouverture de nouveaux marchés et l'établissement de nouvelles structures organisationnelles. L'objectif de l'innovation est de maximiser la rentabilité de la firme innovante, alors que son incidence économique est, selon les mots de Schumpeter, "la destruction créatrice». L'analyse de l'innovation au sein du secteur privé s'est par conséquent concentrée sur les compagnies ou les secteurs de l'économie qui innovent avec le plus de succès et où la destruction créatrice est la plus apparente. Peu importe l'époque étudiée, le degré d'innovation entre les différents secteurs est inégal. Aux États-Unis, par exemple, le secteur de l'automobile essaie de se mesurer aux fabricants les plus avant-gardistes au monde qui sont à l'étranger; le secteur bancaire tente toujours de rattraper les pertes causées par des innovations destructrices comme les swaps sur défaillance et les obligations structurées adossées à des emprunts; le secteur immobilier est toujours plombé par un parc important de biens invendus et invendables; le secteur de la santé se débat avec la limitation des coûts. 
Le secteur des technologies de l'information, dans sa définition large, est généralement perçu comme étant le secteur le plus innovant de l'économie américaine. Il en est ainsi depuis plus de deux décennies, mais avec une intensité variable au fil du temps. Il y a un peu plus de dix ans, pendant la vague d'entreprises Internet naissantes, l'attention était majoritairement portée sur les jeunes entrepreneurs qui tentaient d'offrir leurs produits et services en ligne pour la première fois. Après l'éclatement de la bulle technologique, le secteur des technologies de l'information s'est consolidé et il est maintenant dominé par une poignée d'entreprises qui ont grandi rapidement et avantageusement : Apple dans la conception de produits électroniques, Amazon dans la vente au détail en ligne, IBM dans les services-conseils, Google dans la recherche et Facebook dans le réseautage social. Les chercheurs en innovation s'intéressent désormais à la façon dont ces entreprises soutiennent l'innovation. Il s'agit d'une question ironique, puisqu'il est généralement admis qu'il y a des entraves à l'innovation dans les grandes sociétés et que les innovations les plus importantes sont le fait de petites entreprises naissantes. Certaines grandes entreprises (Amazon, Google, Facebook) sont de jeunes pousses qui ont grandi. La vraie question est de savoir comment elles peuvent continuer à être novatrices.

Deux ouvrages d'intérêt général sur l'innovation publiés récemment et ayant attiré l'intérêt du public sont révélateurs de cette attention de plus en plus portée sur l'innovation au sein des grandes entreprises. The Idea Factory: Bell Labs and the Great Age of American Innovation, écrit par Jon Gertner en 2012, est l'histoire de Bell Laboratories, la division « recherche » de l'entreprise monopolistique de téléphonie américaine (du moins jusqu'à la moitié des années 1980), American Telephone and Telegraph. Imagine: How Creativity Works (2012) de Jonah Lehrer est un résumé de recherches universitaires sur la créativité individuelle et l'innovation organisationnelle, un livre qui s'adresse au grand public ${ }^{2}$. En revanche, l'analyse universitaire de l'innovation la plus citée ${ }^{3}$ au sein du secteur privé est l'article publié par Kanter en 2000.

Gertner raconte l'histoire de Bell Laboratories depuis leur fondation dans les années 1920. Il souligne certaines inventions majeures qui ont vu le jour en leurs murs (le transistor, la théorie de l'information) et présente les inventeurs qui leur sont associés (John Bardeen, Walter Brattain et William Shockley, qui ont reçu le prix Nobel de physique en 1956 pour le transistor; Claude Shannon, universellement reconnu comme le père fondateur de la théorie de l'information). Dans la conclusion, Gertner tente d'expliquer le succès de Bell Laboratories. Il l'attribue à la disponibilité du financement pour les chercheurs, à la culture organisationnelle qui encourageait le partage interdisciplinaire d'idées (par exemple en répartissant les chercheurs de différentes disciplines dans les bureaux donnant sur un long corridor), à la volonté de soutenir la recherche à long terme sur une idée prometteuse et à la volonté d'appuyer la recherche fondamentale potentiellement perturbatrice et non seulement la recherche appliquée.

Lehrer a associé la recherche neurologique sur la créativité à la recherche comportementale sur des organismes novateurs. Par exemple, la recherche neurologique sur la créativité a montré l'importance de la rêverie, de la contemplation et de l'association libre. L'auteur

2 Après publication, il a été découvert que dans l'analyse de la créativité de Lehrer, celui-ci attribuait à l'auteurcompositeur Bob Dylan plusieurs affirmations sur l'origine du classique Like a Rolling Stone qu'on ne retrouvait nulle part dans la littérature sur Bob Dylan recensée par Lehrer. Aucune allégation similaire, par contre, n'a été faite sur les parties du livre de Lehrer qui sont citées dans cet article.

3 Plus de 1207 citations dans Google Scholar (page consultée le 27 avril 2013). 
souligne que 3M et Google fournissent des installations dans leurs campus - une appellation qui illustre bien leur culture - pour la contemplation et le jeu et qu'ils ont pour habitude d'offrir à leurs employés une journée par semaine (ce que Google appelle Innovation Time Off, ou congé pour l'innovation) pour qu'ils s'adonnent à leurs intérêts intellectuels personnels. Lehrer a aussi traité du design de l'espace de travail chez Pixar réalisé par Steve Jobs, espace conçu de façon à encourager les collaborations intellectuelles en plaçant de nombreux services communs - boîtes de courrier, cafétéria, voire salles de toilettes - dans un grand atrium central. Lehrer voulait également découvrir de quelle façon les concentrations d'employés spécialisés et de professionnels, par exemple dans la Silicon Valley, encouragent l'éclosion d'entreprises technologiques. Il a remarqué que l'environnement légal de la Californie favorise la mobilité des travailleurs, car les ententes de non-concurrence et de confidentialité n'y sont pas appliquées.

Une autre approche possible pour stimuler l'innovation utilisée dans le secteur privé est l'innovation ouverte. Il s'agit d'inviter des communautés externes à aider une compagnie à résoudre des problèmes. L'approche de l'innovation ouverte demande une volonté de la part des dirigeants à rendre publics des renseignements exclusifs afin d'augmenter substantiellement le nombre de personnes qui réfléchissent à un problème. L'innovation ouverte peut être mise en œuvre sur le site Web de la compagnie ou par un fournisseur de services d'innovation ouverte tel qu'InnoCentive. Les «têtes chercheuses", pour employer leur terminologie, offrent des récompenses pour la meilleure solution: un rapide survol d'InnoCentive montre que la plupart des récompenses tournent autour des 10000 \$, mais sont à l'occasion aussi élevées que 50000 \$ ou 100000 \$. Les étudiants en créativité suivent un principe lequel les gens qui appliquent une perspective disciplinaire totalement nouvelle sur un problème peuvent avoir plus de succès que ceux qui le connaissent par cœur. L'innovation ouverte, qu'elle soit hébergée sur des sites Internet publics comme InnoCentive ou sur un réseau intranet, pourrait également être utilisée au sein de grandes entreprises où l'une des conséquences de leur taille est le fait que les employés ne peuvent pas connaître autrement le nombre de défis d'innovation avec lesquels l'entreprise doit composer.

\section{L'INNOVATION DU SECTEUR PUBLIC DANS UN CONTEXTE DE DÉPENSES DE RELANCE ET D'AUSTÉRITÉ}

L'innovation dans le secteur privé, qui est fortement axée sur la conception et la mise en marché de nouveaux produits, n'est que partiellement pertinente pour le secteur public. Le rôle du secteur public est principalement d'assurer la prestation de services publics. L'innovation dans ce secteur concerne la plupart du temps de nouvelles méthodes de prestation de ces mêmes services. À l'occasion, le secteur public lance de nouveaux programmes qui pourraient être considérés comme analogues aux nouveaux produits offerts par le secteur privé. Mais l'expérience d'entreprises privées qui ont bâti une culture encourageant l'innovation peut servir aux organismes publics qui tentent de faire de même.

La stimulation économique en réponse à la crise économique mondiale et l'austérité budgétaire pour réduire les dépenses gouvernementales sont deux politiques notables des cinq dernières années qui ont eu des effets mixtes sur l'innovation au sein du secteur public. L'objectif de la première politique était de dépenser rapidement, en particulier dans les secteurs où les ressources étaient limitées, notamment dans la construction. Les travaux prêts à démarrer sont devenus monnaie courante. L'innovation dans le secteur public, qu'elle concerne la manière dont les services publics doivent être offerts ou la création de 
l'infrastructure sociale qui peut être employée d'une façon nouvelle, prend du temps. Par exemple, dans la politique de stimulation économique du gouvernement canadien (Plan d'action économique) figuraient des éléments tels que des subventions pour des rénovations domiciliaires, l'asphaltage d'une ligne de chemin de fer inutilisée pour la transformer en piste cyclable et le remplacement d'un établissement d'enseignement construit en 2004 par un autre établissement inauguré en 2011, celui-là en utilisant des technologies plus vertes. Les citoyens de la plupart des pays ont certainement vécu des expériences semblables. Ce serait un projet de recherche très intéressant que d'échantillonner les politiques de stimulation économique internationalement pour découvrir combien d'innovations, que ce soit à l'intérieur ou hors du secteur public, elles ont permis de réaliser. Mon impression est la suivante: "pas beaucoup », car l'urgence l'aura probablement emporté sur d'autres variables.

En dépit de la fin des politiques de dépenses de relance, la plupart des gouvernements se sont retrouvés aux prises avec des déficits importants en raison du coût continu des renflouements, en particulier des sociétés de services financiers, décidés pendant la crise financière, de l'intérêt sur la dette encourue par la stimulation économique et de la perte de revenus compte tenu du chômage persistant4. Ainsi, la priorité s'est déplacée vers les programmes d'austérité pour éliminer les déficits. Les agences de notation et les marchés des obligations ont tous deux augmenté la pression sur les États déjà aux prises avec de fortes dettes pour qu'ils éliminent leurs déficits. Il peut y avoir certains désaccords entre la gauche et la droite au sujet de la vitesse et de la nature des coupes à réaliser, mais celles-ci demeurent une priorité pour les deux camps.

Comment l'austérité affectera-t-elle l'innovation dans le secteur public? Cela dépendra probablement de l'idéologie politique du gouvernement, de la proximité de la date à laquelle les coupes entreront en vigueur et de l'ampleur des compressions à réaliser. Les gouvernements de droite ont fort probablement leur propre liste de programmes qu'ils souhaitent éliminer pour des raisons idéologiques, et les programmes d'austérité leur fournissent l'occasion de le faire. Les gouvernements de gauche auront une liste plus courte, et leur priorité sera de trouver des moyens moins coûteux pour continuer d'atteindre les buts pour lesquels les programmes existants ont été conçus. Si l'échéancier est court, comme dans le cas d'une politique de dépenses de relance, il y a peu de place pour les coupes novatrices; la priorité est de trouver les programmes que le gouvernement peut immédiatement éliminer. L'ampleur des coupes à effectuer fonctionnerait de manière inverse, dans le sens où une fois toutes les coupes « par décret » faites, les gouvernements commencent à envisager des solutions plus radicales.

Geoff Mulgan (2012), un ancien conseiller en matière d'innovation pour le gouvernement britannique et maintenant cadre supérieur de NESTA, une organisation dont l'objectif est de favoriser l'innovation, contrebalance les coupes budgétaires traditionnelles (cesser certaines activités, geler les salaires, offrir des indemnisations pour les départs volontaires, congédier du personnel, arrêter le recrutement, retarder les dépenses d'investissement) par des innovations organisationnelles (réaliser des économies d'échelle dans les activités gouvernementales, réaliser des économies de diversification, restructurer les procédés de production, par

4 Dans certains cas, comme dans celui du programme TARP du gouvernement américain, une portion substantielle des fonds de renflouement accordés au secteur financier a été remboursée au gouvernement, mais seule une infime partie des fonds de renflouement accordés aux institutions financières de la zone euro sera effectivement récupérée. 
exemple pour éliminer les coûts liés à la réparation d'erreurs en faisant bien les choses dès la première fois, décentraliser la responsabilité par la coproduction, les partenariats avec la société civile ou en faisant appel à des bénévoles, réduire les coûts en augmentant la transparence en matière de dépenses publiques). Bien entendu, les changements organisationnels dont traite Mulgan supposent d'utiliser l'innovation pour répondre à l'austérité. La question est de savoir si les responsables politiques préfèrent des coupes traditionnelles ou novatrices, et à quelle vitesse ils veulent que les coupes soient réalisées ${ }^{5}$. Les innovations organisationnelles proposées par Mulgan ne peuvent pas être exécutées instantanément, car elles impliquent des étapes telles que l'analyse et la reconfiguration des opérations et la négociation de partenariats externes.

\section{UNE RECHERCHE BASÉE SUR UN PRIX RÉCOMPENSANT L'INNOVATION}

Depuis plus de deux décennies, nous poursuivons une recherche en utilisant des dossiers de candidature à un prestigieux prix d'innovation en gestion publique, l'Innovations in American Government Awards Program de la Harvard Kennedy School (Borins, 1998 et 2006). Cette recherche fournit un angle privilégié pour étudier le processus de l'innovation dans le secteur public et pour anticiper de futures innovations. Ce programme de prix annuel est ouvert à tous les secteurs politiques et à tous les ordres de gouvernement et reçoit normalement 500 candidatures initiales, lesquelles sont évaluées sur la base des réponses apportées à un questionnaire court demandant une description du programme et des preuves de la façon dont il respecte les critères du prix, soit la nouveauté, l'efficacité, la portée et la transférabilité. Environ 150 demi-finalistes sont choisis par des juges experts qui les invitent à constituer un dossier complet dans lequel ils doivent détailler les origines, l'évolution, le budget, la structure organisationnelle, les résultats et la reconnaissance du programme. Les dossiers complets des demi-finalistes comptent une douzaine de pages. Le codage de ces documents exhaustifs a permis de brosser un portrait détaillé du processus d'innovation dans le secteur public. Une recherche précédente (Borins, 1998 et 2006) a porté sur 217 dossiers de demi-finalistes déposés entre 1990 et 1994.

Notre recherche actuelle porte sur un échantillon de 234 demandes initiales et sur chacun des 127 dossiers des demi-finalistes au programme de prix de 2010. Ainsi, les données recueillies peuvent être utilisées pour montrer comment l'innovation dans la gestion publique a changé et comment elle est demeurée constante pendant presque vingt ans. Le codage des 234 demandes initiales et des 127 dossiers des demi-finalistes a été réalisé, avec un ratio d'uniformité entre les codeurs de $90 \%$. Ce protocole de recherche peut paraître opportuniste, car un questionnaire conçu pour un seul usage (une compétition en vue de recevoir un prix) a été utilisé dans un autre but, celui d'étudier le processus d'innovation. En ayant recours à des juges experts et en procédant à une vérification des innovations qui se rendent à la ronde finale, le programme décourage l'exagération de la part des candidats, et de ce fait augmente la fiabilité de la recherche basée sur leurs dossiers de candidature ${ }^{6}$

5 Le gouvernement Harper, dans son budget 2012, a effectué les principales compressions (près de $7 \%$ ) dans les budgets discrétionnaires ministériels. Dans le budget, on supposait qu'une grande partie de l'épargne serait réalisée par des coupes dans les services administratifs et par l'utilisation des technologies de l'information (des coupes novatrices, selon la terminologie de Mulgan), mais dans la pratique, elles se concentrent plutôt dans les réductions de programmes.

6 Les critères et le processus de sélection des prix font en sorte que les innovations choisies comme demifinalistes ont fort probablement réussi à fournir des avantages aux citoyens et à demeurer en activité. Les innovations anciennes ne peuvent pas être soumises, et il est peu probable que les programmes défaillants soient 
(Borins, 1998, p. 17-18). Dans cette section, les résultats préliminaires de cette recherche sont présentés, résultats qui seront par la suite publiés dans un ouvrage (titre provisoire : The Persistence of Innovation) par Brookings Institute Press. Les questions utilisées pour décrire les résultats présentés dans les tableaux suivants sont fournies en note de bas de page. En utilisant les réponses codées, il est également possible d'isoler les facteurs exogènes influant sur l'innovation dans le secteur public et de faire des prévisions au sujet de ces facteurs et de la façon dont ils affecteront la nature et la portée de l'innovation dans le secteur public, comme nous le verrons plus loin.

\section{TABLEAU 1 : LES CARACTÉRISTIQUES DES INNOVATIONS7}

\begin{tabular}{|c|c|c|c|c|}
\hline Caractéristique & $\begin{array}{c}\text { Demandes } \\
\text { initiales de } 2010\end{array}$ & $\begin{array}{l}\text { Demandes des non- } \\
\text { demi-finalistes de } \\
2010\end{array}$ & $\begin{array}{l}\text { Demi-finalistes } \\
\text { de } 2010\end{array}$ & $\begin{array}{l}\text { Demi-finalistes } \\
\text { de } 1990 \text { à } 1994\end{array}$ \\
\hline $\begin{array}{l}\text { Collaboration au sein } \\
\text { du gouvernement }\end{array}$ & $58 \%$ & $58 \%$ & $59 \%$ & $21 \%$ \\
\hline Collaboration externe & $65 \%$ & $63 \%$ & $67 \%$ & $28 \%$ \\
\hline $\begin{array}{l}\text { Technologie de } \\
\text { l'information }\end{array}$ & $36 \%$ & $35 \%$ & $37 \%$ & $28 \%$ \\
\hline $\begin{array}{l}\text { Amélioration du } \\
\text { processus }\end{array}$ & $29 \%$ & $30 \%$ & $28 \%$ & $34 \%$ \\
\hline Habilitation du citoyen & $16 \%$ & $22 \%$ & $14 \%$ & $26 \%$ \\
\hline $\begin{array}{l}\text { Utilisation de } \\
\text { bénévoles }\end{array}$ & $13 \%$ & $16 \%$ & $10 \%$ & $7 \%$ \\
\hline $\begin{array}{l}\text { Utilisation d'incitatifs } \\
\text { économiques }\end{array}$ & $10 \%$ & $7 \%$ & $13 \%$ & $8 \%$ \\
\hline $\begin{array}{l}\text { Changements dans } \\
\text { l'attitude du public }\end{array}$ & $11 \%$ & $12 \%$ & $9 \%$ & $13 \%$ \\
\hline $\mathrm{N}$ & 234 & 126 & 108 & 217 \\
\hline
\end{tabular}

Source : Pour les demi-finalistes de 1990 à 1994, Borins (1998), tableau 2.1 (p. 20) et tableau 2.2 (p. 26).

Le tableau 1 montre les caractéristiques fondamentales des demandes initiales aux Innovations Awards de 2010, et les compare à l'échantillon des demi-finalistes de 1990 à 1994 utilisé dans Innovating with Integrity (Borins, 1998). Comme le codage permettait des caractéristiques multiples pour chaque innovation, les pourcentages totalisent plus de 100 . Deux observations ressortent. L'incidence des caractéristiques pour les demi-finalistes de 2010 et les non-demi-finalistes est très semblable, une différence de 1 ou $2 \%$ tout au plus la

soumis. Ainsi, cette méthodologie de recherche ne détectera pas les innovations non réussies. Étant donné la réticence normale à discuter des échecs, il est peu plausible que n'importe quelle méthodologie volontaire de recherche de ce type détecterait les innovations non réussies. Le journalisme d'enquête et les commissions d'enquête pourraient sans doute mieux atteindre cet objectif, mais ils fournissent des études de cas plutôt que des bases de données utilisables pour l'analyse quantitative.

7 Tableau 1 : Formulaire de demande initiale : « Résumez en deux phrases votre innovation. Cette description doit être précise et transmettre de façon succincte l'essence de votre innovation. » Dossier de candidature des demifinalistes: "Décrivez votre innovation. Quel problème votre innovation vient-elle résoudre? De quelle façon exactement votre programme ou votre politique sont-ils novateurs? De quelle façon votre innovation a-t-elle changé les anciennes façons de faire? » 
plupart du temps. En comparant les demandes de 2010 à celles de 1990 à 1994, on note une incidence nettement plus élevée de nos jours au chapitre de la collaboration interorganisationnelle, soit à l'intérieur du gouvernement (58\%) ou entre le gouvernement et la société civile (65\%); cette collaboration était respectivement de 20 et de $29 \%$ il y a deux décennies. Par collaboration interorganisationnelle à l'intérieur du gouvernement, nous désignons la collaboration entre des organismes d'un même ordre de gouvernement ou entre des organismes de différents ordres gouvernementaux. Par collaboration interorganisationnelle avec la société civile, nous faisons référence à la collaboration entre les organismes gouvernementaux et le secteur privé ou le secteur sans but lucratif. Pour les autres caractéristiques, les écarts entre les demandeurs de 2010 et ceux de 1990 à 1994 ne dépassent pas $10 \%$.

La collaboration interorganisationnelle est devenue une matière importante de la recherche dans l'administration publique, qui embrasse à la fois les études sur la collaboration au sein du secteur public (Bardach, 1998 et 2008) et celles sur la collaboration entre le secteur public et la société civile (Donahue et Zeckhauser, 2011; Goldsmith et Eggers, 2004). Dans le contexte de l'innovation, cela signifie que des gestionnaires publics novateurs sont maintenant plus fréquemment impliqués dans l'élaboration de solutions à des problèmes difficiles, voire épineux, qui dépassent les frontières organisationnelles; il y a vingt ans, ils lançaient plus fréquemment des initiatives pour améliorer l'efficacité ou l'efficience de leur propre organisme. Le fait que l'incidence de la collaboration soit sensiblement la même pour les demi-finalistes et les non-demi-finalistes signifie que les juges ne favorisaient pas ceux qui optaient pour la collaboration.

D’autres caractéristiques observées presque aussi fréquemment chez les demandeurs de 2010 et ceux du début des années 1990 étaient l'amélioration de processus, l'utilisation des technologies de l'information et l'habilitation des citoyens. Les retombées futures de toutes ces caractéristiques devraient changer la nature de l'innovation au sein du gouvernement à l'avenir. Les technologies de l'information évolueront rapidement et les nouvelles technologies telles que l'infonuagique et la connexion à Internet de toute sorte de machines, de dispositifs et d'appareils («Internet des choses ») sont susceptibles de trouver des usages dans le secteur public. Nous pouvons espérer voir une certaine utilisation de l'innovation ouverte dans le secteur public. Le gouvernement des États-Unis a pris l'initiative sur son site Web qui montre qu'environ 300 concours sont actuellement ouverts ${ }^{8}$. Dans un autre type d'innovation ouverte, le gouvernement ne demande pas de solutions spécifiques, mais rend plutôt ses bases de données disponibles aux citoyens et aux développeurs Web qui créeront de nouvelles applications. Cette façon de faire a été mise en œuvre pour la première fois par le district de Colombia (ce qui lui a valu un prix Innovation in American Government en 2009), et fut rapidement aoptée par le gouvernement fédéral. Les sites de réseautage social sont maintenant devenus un lieu important pour les initiatives d'habilitation du citoyen lancées par le gouvernement, les partis politiques ou les lobbys de la société civile.

L'amélioration du processus est un thème commun à beaucoup d'innovations. Plusieurs éléments peuvent intervenir dans une initiative d'amélioration de processus, y compris la

8 Le site est accessible à l'adresse suivante: www.challenge.gov. Ces concours sont d'importance variable. Les plus petits concernent la création de véhicules de communication tels que des sites Web ou des campagnes de marketing social. Ceux-ci pourraient être considérés comme analogues à l'externalisation, sauf que plutôt que d'accorder le contrat à la proposition gagnante, le gouvernement demande à tous les soumissionnaires de réaliser le projet, puis choisit le meilleur. Quoi qu'il en soit, le nombre d'activités sur le site indique que cette approche a un potentiel considérable (De Souza, 2012). 
cueillette et l'analyse des données produites à l'interne et liées au processus de prestation de services, la mise en œuvre de certaines idées de restructuration présentées par Mulgan et l'application de découvertes récentes issues de la psychologie appliquée (comme l'utilisation de l'option d'exclusion plutôt que d'inclusion en matière d'exemples de comportements publics moraux). L'analyse des données comme méthode pour améliorer le rendement est née dans la Ville de New York, d'abord dans le domaine du maintien de l'ordre, mais a été reprise par beaucoup d'autres gouvernements. L'Office of Policy and Strategic Planning de la Ville de New York est maintenant un chef de file dans l'analyse de métadonnées sur l'amélioration de la prestation de services et l'efficacité de la normalisation (Feuer, 2013; Lohr, 2013). Les cabinets-conseils considèrent le secteur public comme un marché où ils ont la possibilité de mettre en pratique leurs capacités à analyser des métadonnées, comme IBM le fait dans la planification d'urgence (inondations, éboulements) à Rio de Janeiro (Singer, 2012). Ces suggestions au sujet de la prochaine vague d'innovation dans le secteur public ne sont pas exhaustives, mais elles illustrent le fait que l'innovation dans le secteur public concerne souvent la mise en œuvre de nouvelles méthodologies et idées utilisées par le privé.

\section{TABLEAU 2 : LES AUTEURS DE L'INNOVATION ${ }^{9}$}

\begin{tabular}{|l|c|c|}
\hline & $\begin{array}{c}\text { Demi-finalistes } \\
\text { de } 2010\end{array}$ & $\begin{array}{c}\text { Demi-finalistes } \\
\text { de } 1990 \text { à } 1994\end{array}$ \\
\hline Politicien & $34 \%$ & $18 \%$ \\
\hline Directeur d'agence & $44 \%$ & $39 \%$ \\
\hline Cadre intermédiaire & $40 \%$ & s. o. \\
\hline Personnel de première ligne & $22 \%$ & S. o. \\
\hline Cadre intermédiaire et/ou personnel de première ligne & $46 \%$ & $48 \%$ \\
\hline Client ou partenaire de programme & $27 \%$ & $2 \%$ \\
\hline Groupe d'intérêt & $11 \%$ & $13 \%$ \\
\hline N & 127 & 217 \\
\hline Source : Pour les demi-finalistes de 1990 à 1994, Borins (1998), tableau 3.1 (p. 39). & \\
\hline
\end{tabular}

La référence aux « héros locaux » dans le sous-titre de l'ouvrage Innovating with Integrity: How Local Heroes are Transforming American Government est liée à l'un des résultats principaux de du livre, à savoir que le personnel de première ligne et les cadres intermédiaires étaient fréquemment les auteurs des innovations. Cette conclusion est surprenante en raison de la nature traditionnellement descendante et politiquement allergique au risque de la gestion publique. En effet, l'esprit d'initiative de la part des fonctionnaires de première ligne et des cadres intermédiaires est devenu partie intégrante du débat sur la nouvelle gestion publique qui faisait rage lors de l'écriture d'Innovating with Integrity. Les critiques de la nouvelle gestion publique (DeLeon et Denhardt, 2000; Terry, 1998) considéraient les fonctionnaires ayant l'esprit entrepreneurial comme étant des électrons libres, des briseurs de règles s'adonnant à des jeux de pouvoir et se faisant de la publicité. À l'inverse, les partisans de la

9 Tableau 2: «Quels individus ou groupes d'individus sont considérés comme étant les auteurs premiers de votre programme?» 
nouvelle gestion publique (Barzelay, 1992; Gore, 1993; Moore, 1995) ont présenté des exemples de fonctionnaires apportant des apports valables et ont argué que le fait de se libérer des contraintes internes aurait comme résultat positif de stimuler l'esprit d'entreprise.

Le tableau 2 illustre le schéma des réponses des demi-finalistes à la question portant sur les auteurs. La conclusion étonnante d'Innovating with Integrity reste applicable aux demifinalistes de 2010, où $46 \%$ des innovations ont été lancées par les cadres intermédiaires ou $\mathrm{du}$ personnel de première ligne. Un pourcentage presque identique à celui des demifinalistes de 1990 à 1994 (48 \%).

Les demi-finalistes de 2010, cependant, indiquent aussi un nombre légèrement plus élevé d'initiatives lancées par des politiciens et des directeurs d'agence et un nombre beaucoup plus élevé de clients ou de partenaires de programme que les demi-finalistes de 19901994. Il en est probablement ainsi en raison du niveau beaucoup plus élevé de collaboration interorganisationnelle montré par les demi-finalistes de 2010. La mention fréquente des clients ou des partenaires d'un programme en tant qu'auteurs de l'innovation est une preuve de cette tendance. En outre, dans des rapports verticaux traditionnels d'autorité, il est difficile pour le personnel de première ligne ou les cadres intermédiaires d'introduire des innovations collaboratives; la collaboration exige habituellement de la part des directeurs d'agence ou des politiciens qu'ils négocient des ententes interorganisationnelles informelles ou des protocoles officiels écrits qui régissent la collaboration entre les organisations.

\section{TABLEAU 3 : LES CONDITIONS MENANT À L'INNOVATION ${ }^{10}$}

\begin{tabular}{|l|c|c|}
\hline & $\begin{array}{c}\text { Demi-finalistes } \\
\text { de } 2010\end{array}$ & $\begin{array}{c}\text { Demi-finalistes } \\
\text { de } 1990 \text { à } 1994\end{array}$ \\
\hline $\begin{array}{l}\text { Influence politique (mesures } \\
\text { législatives ou lobbyisme) }\end{array}$ & $41 \%$ & $19 \%$ \\
\hline Crise, actuelle ou anticipée & $14 \%$ & $30 \%$ \\
\hline Problème à régler & $74 \%$ & $49 \%$ \\
\hline Occasion & $20 \%$ & $33 \%$ \\
\hline N & 127 & 217 \\
\hline
\end{tabular}

Source : Pour les demi-finalistes de 1990 à 1994, Borins (1998), tableau 3.2 (p. 41).

Le tableau 3 montre les principales conditions qui mènent à l'innovation dans le secteur public. Ainsi, les innovations sont plus fréquemment des tentatives pour résoudre des problèmes que des réponses à une crise. Ceci contredit l'opinion cynique qu'on se fait du secteur public, ancrée dans la théorie du choix public, selon laquelle les ministères ou les agences, parce qu'ils sont souvent des monopoles, feraient fi des problèmes jusqu'à ce qu'ils se soldent en échecs de performance dramatiques ayant de grandes répercussions (Borins, 1998). Les innovations surviennent de la résolution de problèmes proactive des fonctionnaires ou des politiciens. Un deuxième constat intéressant est que beaucoup d'innovations sont le résultat d'occasions favorables. En plus de nouvelles technologies, ces occasions favorables font parfois émerger de nouvelles sources de revenus. Un avantage inattendu mentionné dans plusieurs dossiers de candidature à l'Innovations in American Government

10 Tableau 3 : «De quelle façon l'initiative représentant votre idée novatrice a-elle été créée et lancée? » 
Awards Program était le Tobacco Master Settlement Agreement de 1998 qui a obligé les fabricants de produits du tabac à payer aux gouvernements étatiques des milliards de dollars pour compenser les coûts des soins en santé engendrés par le tabagisme.

Pour l'avenir, nous voudrions prévoir comment ces conditions évolueront et quelles sortes d'innovations il en résultera. Les problèmes majeurs auxquels le gouvernement doit faire face ne manquent pas : changements démographiques et vieillissement de la population, disponibilité réduite des ressources non renouvelables, changement climatique. Les innovations tireront également profit de nouvelles possibilités, en particulier celles créées par les nouvelles technologies et les avantages fiscaux inattendus.

Les questionnaires sur l'innovation portaient également sur les obstacles rencontrés lors de la mise en œuvre d'une innovation. Les trois principaux obstacles rapportés, dans des fréquences relativement semblables pour les demi-finalistes de 2010 et ceux de 1990 à 1994, étaient la résistance bureaucratique, les problèmes logistiques et le besoin de ressources financières, chacun constituant environ $20 \%$ de tous les obstacles cités (Borins, 1998, p. 66-71). Des problèmes logistiques ont souvent pu être résolus avec des solutions de rechange et une gestion créative des ressources. La réponse à la résistance bureaucratique a demandé la mise en œuvre de tactiques de changements organisationnels tels que la consultation, la cooptation, la compensation et la formation. Trouver des ressources financières était l'écueil le plus difficile à surmonter. Les solutions visaient à diversifier les sources de financement, par exemple en trouvant du financement de programmes dans les secteurs privé ou sans but lucratif, en se contentant de ce qui était disponible et en persistant dans la recherche de fonds (Borins, 1998, p. 71-79).

Le tableau 4 fait état de l'étendue de la diversité des sources de financement (de même que des mesures de l'étendue de la collaboration interorganisationnelle) pour les demifinalistes de 2010. Il compare l'ordre de gouvernement de l'agence faisant la demande aux ordres de gouvernement et aux organismes non gouvernementaux cités comme ayant contribué à son financement. Environ $10 \%$ des demandes ont été faites pour des programmes du gouvernement fédéral, $40 \%$ pour des programmes de gouvernements étatiques et $50 \%$ pour des programmes de gouvernements municipaux. Tandis que $10 \%$ des demifinalistes étaient des programmes du gouvernement fédéral, celui-ci a été enregistré comme source de financement dans $45 \%$ des dossiers; les gouvernements étatiques et municipaux ont tous deux été enregistrés comme source de financement dans $47 \%$ des demandes. Comme $52 \%$ des demi-finalistes étaient issus de gouvernements municipaux, cela signifie que $5 \%$ des programmes de gouvernements municipaux ont reçu leur financement entièrement de sources autres que du gouvernement municipal. En outre, $24 \%$ des demifinalistes ont reçu des fonds du secteur privé et $17 \%$ d'organismes sans but lucratif, surtout de fondations. En tout, $13 \%$ ont reçu leur financement à partir des frais d'utilisation. La somme des pourcentages dans cette colonne est de $193 \%$, indiquant que la demande moyenne a reçu du financement de deux sources. La dernière colonne montre également le pourcentage moyen du budget des demi-finalistes fourni par chaque source de financement. Ces moyennes varient de $27 \%$ pour les organismes sans but lucratif à $64 \%$ pour les gouvernements municipaux. Ainsi, les diverses sources de financement n'allouent pas de montants symboliques, mais accordent plutôt un financement important aux demi-finalistes. 


\section{TABLEAU 4： LES SOURCES DE FINANCEMENT POUR LES DEMI-FINALISTES DE $2010^{11}$}

\begin{tabular}{|l|c|c|c|}
\hline Source de financement & $\begin{array}{c}\text { Ordre de } \\
\text { gouvernement } \\
\text { posant sa candidature }\end{array}$ & $\begin{array}{c}\text { Candidatures } \\
\text { recevant du } \\
\text { financement }\end{array}$ & $\begin{array}{c}\text { Pourcentage moyen du } \\
\text { budget fourni par une } \\
\text { source }\end{array}$ \\
\hline Gouvernement fédéral & $9 \%$ & $45 \%$ & $56 \%(48)$ \\
\hline Gouvernement étatique & $39 \%$ & $47 \%$ & $54 \%(50)$ \\
\hline Gouvernement municipal & $52 \%$ & $47 \%$ & $64 \%(50)$ \\
\hline Secteur privé & & $24 \%$ & $41 \%(25)$ \\
\hline Sans but lucratif & & $17 \%$ & $27 \%(12)$ \\
\hline Frais d'utilisation & 127 & $13 \%$ & $51 \%(13)$ \\
\hline N & & 127 &
\end{tabular}

Note: Les nombres entre parenthèses dans la dernière colonne indiquent le nombre d'observations utilisées pour calculer le pourcentage moyen du budget fourni par chaque source de financement.

\section{TABLEAU 5: LES LACUNES IMPORTANTES SUBSISTANTES DE L'INNOVATION ${ }^{12}$}

\begin{tabular}{|l|c|c|}
\hline A besoin d'être peaufiné & $\begin{array}{c}\text { Demi-finalistes } \\
\text { de } 2010\end{array}$ & $\begin{array}{c}\text { Demi-finalistes } \\
\text { de } 1990 \text { à } 1994\end{array}$ \\
\hline A besoin de financement & $41 \%$ & $29 \%$ \\
\hline Ne s'est pas diffusé comme escompté & $33 \%$ & $39 \%$ \\
\hline A de la difficulté à entretenir la collaboration & $20 \%$ & $12 \%$ \\
\hline N & $5 \%$ & $5 \%$ \\
\hline
\end{tabular}

Source : Pour les demi-finalistes de 1990 à 1994, Borins (1998), tableau 4.5 (p. 83).

Les innovateurs américains ont été interrogés sur les lacunes persistantes de leur programme. Le tableau 5 présente leurs réponses. Le besoin de peaufinage peut être vu comme un problème logistique continu, et le manque de ressources demeure un fardeau pour un grand nombre d'innovateurs. Cette conclusion nous ramène à un point clé concernant l'innovation, à savoir qu'elle exige des ressources financières. Ce fait a été clairement démontré par des chefs de file de l'innovation du secteur privé, par exemple Bell Laboratories qui fournit des ressources internes de façon stable à ses chercheurs, et des compagnies comme $3 \mathrm{M}$ et Google qui donnent au personnel de recherche, de développement et d'ingénierie un jour par semaine pour poursuivre leurs propres activités intellectuelles. En revanche, l'innovation dans les entreprises naissantes de Silicon Valley est financée par des spécialistes du capital de risque. L'innovation dans le secteur public a également besoin de financement. Il peut parfois provenir des recettes fiscales normales, et parfois, de sources de financement inattendues comme le Tobacco Master Settlement Agreement. Dans d'autres cas, les ressources pour soutenir l'innovation ont été prises à même des surplus amassés par des gestionnaires futés du secteur public. Les coupes budgétaires traditionnelles, dans

11 Tableau 4: "Quel est le budget de fonctionnement actuel de votre programme? Quelles sont les sources de financement de votre programme (p. ex. fonds municipaux, étatiques, fédéraux, privés)? Quel pourcentage des revenus annuels chaque source représente-t-elle? »

12 Tableau 5 : «Qu'est-ce qui, selon vous, constitue la lacune persistante de votre programme? » 
un premier temps, éliminent habituellement les ressources excédentaires. Les surplus cachés qui pourraient être employés pour soutenir la recherche sont rendus à l'agence de contrôle des dépenses. Quand tout le personnel disponible doit travailler plus fort, par exemple en raison du non-remplacement de travailleurs, toute initiative du même type que l'Innovation Time Off de Google disparaît.

\section{CONCLUSION}

Cet article a présenté un portrait de l'innovation dans la gestion publique ${ }^{13}$. Ce portrait s'est montré constant aux États-Unis pendant plus de deux décennies pour plusieurs des variables du processus, telles que l'auteur de l'innovation, les conditions menant à l'innovation, ses obstacles et la façon dont ils ont été surmontés, et les lacunes subsistantes de l'innovation. Les données indiquent également une évolution, en particulier en ce qui concerne la plus grande prépondérance de la collaboration au sein du gouvernement et entre le public et les secteurs privé ou sans but lucratif.

Du point de vue de la prévision des tendances de l'innovation dans le secteur public, deux variables principales sont à considérer : les problèmes anticipés auxquels le secteur public doit répondre et l'évolution dans les caractéristiques définissant l'innovation, soit le matériel mis à la disposition des innovateurs.

Tandis que les dossiers de candidature pour des prix d'innovation nous permettent d'élaborer des portraits de programmes novateurs, ils n'éclairent pas beaucoup sur les ministères et les agences qui ne soumettent pas de demandes. Nous voudrions voir l'esprit d'innovation envahir le gouvernement jusqu'au plus haut niveau possible. Cela supposerait d'encourager le type d'innovations présentées ici. Ainsi, si la collaboration interorganisationnelle est présente dans un pourcentage aussi élevé dans les dossiers de candidature, il importe que les règles et les pratiques au sein du gouvernement l'encouragent. Si l'ouverture aux individus et aux groupes à l'extérieur du gouvernement peut contribuer à résoudre des problèmes, alors les règles et les pratiques du secteur public devraient favoriser l'innovation ouverte version secteur public. L'importance de la lutte que beaucoup d'innovateurs mènent pour trouver des ressources suggère que l'innovation dans le secteur public pourrait être augmentée par la création de capital de risque analogue à celui du secteur privé.

La tendance répandue à l'austérité aura des conséquences importantes sur l'innovation dans le secteur public. D'une part, elle met de la pression sur le gouvernement pour qu'il restructure ses services de manière créative. D'une autre part, elle peut amputer les ressources nécessaires pour soutenir les innovations qui rendraient ces restructurations possibles. Les agences centrales gérant les programmes d'austérité devront relever le défi de combiner la pression de restructurer en fournissant à tout le moins quelques ressources additionnelles pour faciliter le processus de restructuration.

En période de crise, il est facile de croire que l'innovation est un luxe, qu'elle est inappropriée, voire non pertinente, aux tâches quotidiennes d'éteindre des feux et de repousser des désastres imminents. Il n'en est rien. L'innovation au sein du gouvernement, en particulier dans ces "périodes intéressantes ", peut être un défi, mais également une nécessité. La question ne doit pas être si on peut y arriver, mais de quelle façon.

13 II a été délibérément choisi de ne pas employer le terme "modèle », parce qu'il a une connotation théorique et il n'a pas encore été testé empiriquement. Dans cette recherche, le portrait est inductif, dérivé des données. Une dénomination plus appropriée serait l'idéal type de Weber. 


\section{BIBLIOGRAPHIE}

Bardach, E. (2008). « Development Processes: A Conceptual Exploration », dans S. Borins (dir.), Innovations in Government: Research, Recognition, and Replication, Washington, Brookings, p. 113-137.

Bardach, E. (1998). Getting Agencies to Work Together: The Practice and Theory of Managerial Craftsmanship, Washington, Brookings.

Barzelay, M. (1992). Breaking Through Bureaucracy: A New Vision for Managing in Government, Berkeley, University of California.

Borins, S. (2006). The Challenge of Innovating in Government, IBM Center for the Business of Government, www.businessofgovernment.org/sites/default/files/BorinsInnovatingInGov.pdf (page consultée en mai 2013).

Borins, S. (1998). Innovating with Integrity: How Local Heroes are Transforming American Government, Washington, Georgetown.

Borins, S. (1988). "Public Choice: "Yes Minister" Made it Popular, but Does Winning a Nobel Prize Make it True? », Canadian Public Administration, vol. 31, n 1, p. 12-26.

DeLeon, L. et R. Denhardt (2000). «The Political Theory of Reinvention », Public Administration Review, vol. 60, n 2, p. 89-97.

De Souza, K. (2012). Challenge.gov: Using Competitions and Awards to Spur Innovation, IBM Center for the Business of Government, www.businessofgovernment.org/sites/default/files/ Challenge.gov.pdf (page consultée en juin 2013).

Donahue, J. D.et R. Zeckhauser (2011). Collaborative Governance: Private Roles for Public Goals in Turbulent Times. Princeton, Princeton University Press.

Feuer, A. (2013). « The Mayor's Geek Squad», The New York Times, 23 mars.

Gertner, J. (2012). The Idea Factory: Bell Labs and the Great Age of American Innovation, New York, Penguin.

Goldsmith, S. et W. Eggers (2004). Governing by Network: The New Shape of the Public Sector, Washington, Brookings Institution Press.

Gore, A. (1993). Creating a Government that Works Better and Costs Less: Report of the National Performance Review, New York, Times Books.

Kanter, R. (2000). "When a Thousand Flowers Bloom », dans R. Swedberg, Enterpreneurship: The Social Science View, Oxford, Oxford University Press, p. 167-210.

Lehrer, J. (2012). Imagine: How Creativity Works, New York, Penguin.

Lohr, S. (2013). «SimCity, for Real: Measuring an Untidy Metropolis », The New York Times, 23 février.

Moore, M. (1995). Creating Public Value: Strategic Management in Government, Cambridge, Harvard University Press.

Mulgan, G. (2012). Austerity and Innovation, Présentation, Toronto, 2 mai.

Singer, N. (2012). « Mission Control, Built for Cities », The New York Times, 3 mars.

Schumpeter, J. (2008). Capitalism, Socialism, and Democracy, 3e éd. New York, Harper Modern Classics [1 re éd. 1943].

Terry, L. (1998). «Administrative Leadership: Neo-Managerialism, and the Public Management Movement », Public Administration Review, vol. 58, n³, p. 194-200. 